\title{
Facteurs Associes a la Qualite des Eaux Consommees dans la Commune d'Abomey-Calavi au Sud du Benin en Afrique de L'Ouest
}

\author{
Hondjenou Martin, \\ Landeou Codjo Rodrigue, \\ Azonhe Thierry, \\ Houssou Sègbè Christophe,
}

Laboratoire Pierre Pagney : Climats, Eaux, Ecosystèmes et Développement,

Département de géographie et Aménagement du Territoire,

Université d'Abomey-Calavi (UAC), Cotonou, République du Bénin

(Afrique de l'Ouest)

URL:http://dx.doi.org/10.19044/esj.2019.v15n30p56

\section{Résumé}

En dépit de l'insuffisance des ouvrages hydrauliques implantés pour l'approvisionnement en eau de boisson, la lutte contre le manque d'eau potable au Bénin est un gage de recul des frontières des maladies hydriques dans toutes les localités du pays comme dans la Commune d'Abomey-Calavi. Ce travail a pour objectif de déterminer la qualité physico-chimique et bactériologique de l'eau consommée et la prévalence des maladies hydriques observées dans la commune d'Abomey-Calavi. Pour y parvenir, l'approche méthodologique consiste en une enquête de terrain auprès de 399 ménages de ladite commune. Ces données ont été complétées par 33 échantillons d'eau prélevés à la source, durant le transport et au stockage dans les arrondissements de la Commune d'Abomey-Calavi. Les paramètres physico-chimiques ( $\mathrm{pH}$, TDS, conductivité, nitrites, nitrates et l'ammonium) et bactériologiques ont été analysés dans tous les échantillons d'eau prélevés. L'eau de boisson est contaminée par les Escherichia coli, les coliformes fécaux et totaux de la source jusqu'au stockage. Le taux de prévalence est estimé à 30,37 cas pour 1000 habitants en 2017. Donc les maladies hydriques constituent d'ores et déjà un véritable problème de santé publique car la norme de l'OMS en la matière est de 0,01 cas pour 1000 habitants. L'amélioration de la qualité de l'eau permettra de réduire considérablement la propagation de ces maladies. Il est donc important de mettre à la disposition des populations des équipements d'approvisionnement en eau potable. 
Mots clés: Commune d'Abomey-Calavi, Eau de boisson, Qualité de l'eau, Maladies hydriques, Bénin

\title{
Factors Associated with the Quality of Water Consumed in Common of Abomey-Calavi South of Benin in West Africa
}

\author{
Hondjenou Martin, \\ Landeou Codjo Rodrigue, \\ Azonhe Thierry, \\ Houssou Sègbè Christophe,
}

Laboratoire Pierre Pagney : Climats, Eaux, Ecosystèmes et Développement, Département de géographie et Aménagement du Territoire, Université d'Abomey-Calavi (UAC), Cotonou, République du Bénin (Afrique de l'Ouest)

\begin{abstract}
In spite of the insufficiency of the village hydraulic works installed for the supply of drinking water, the fight against the lack of drinking water in Benin is a guarantee of receding the borders of the water-related diseases in all the localities of the country as in the Commune of Abomey-Calavi. The objective of this work is to determine the water quality and the prevalence of water-borne diseases in this community. To achieve this, the methodological approach consists of a field survey among 399 households of the commune. These data were complemented by 33 water samples taken at source, during transport and storage in the boroughs of the municipality of Abomey-Calavi. The physic chemical parameters ( $\mathrm{pH}$, TDS, conductivity, nitrites, nitrates and ammonium) and bacteriological parameters were analyzed in all water samples taken. The drinking-water is contaminated by Escherichia coli, of total and total coliforms at the source were counted in the water. This prevalence is 30.37 cases per 1,000 inhabitants in 2017. Thus, water-bornel diseases are already a real public health problem because the WHO standard for this is 0.01 cases per 1000 inhabitants. Improvement in water quality, sanitation, and personal hygiene will make it possible to reduce considerably the propagation of these diseases and several others. It is therefore important to provide these populations with the necessary equipment for an adequate drinking water.
\end{abstract}


Keywords: Benin, Drinking water, Commune of Abomey-Calavi, Waterborne diseases, Water quality

\section{Introduction}

L'eau est une ressource aussi précieuse que vitale et les hommes doivent en disposer en quantité' et en qualité. L'accès à une eau de boisson saine est incontestablement bénéfique pour la santé. Dans le monde, 1,1 milliard de personnes n'ont pas un accès suffisant à l'eau potable (CAE, 2010). Lorsqu'elle est accessible, l'eau est souvent sujette à une contamination chimique et/ou bactériologique (Sohounou, 2018). La contamination fécale de l'eau de consommation est d'origine humaine ou animale, directe ou indirecte (Lalèyê, 2016). L'utilisation d'une telle eau comme boisson ou dans la préparation des aliments peut être à l'origine de nouveaux cas d'infections. Les agents pathogènes en cause sont des bactéries, des virus et des protozoaires (Lègba, 2017). Ils peuvent provoquer des maladies de gravité variable, de la gastro-entérite bénigne a la dysenterie, à l'hépatite ou à la fièvre typhoïde, voire à la diarrhée sévère et parfois mortelle (Sèbo, 2014). Les maladies d'origine hydrique entraineraient ainsi chaque année 3,4 millions de décès, dont 2,2 millions par maladies diarrhéiques, notamment le cholera (OMS, 2016). Au Benin, la quantité utilisée par personne et par jour est de 60 litres pour chaque citadin et de dix à 20 litres pour chaque habitant en milieu rural (Degbey et al 2011). En effet, le déficit en eau de consommation est estime à 59 \% (DG-Eau, 2010). La société nationale des eaux du Bénin capable d'effectuer un traitement efficace de l'eau dessert quasi exclusivement les zones urbaines. Abomey-Calavi, une ville semi-urbaine jouxtant Cotonou, la capitale économique, a connu un accroissement rapide de la population qui est passée de 307745 habitants en 2002 à 656358 habitants en 2013 (INSAE, 2013). Cet accroissement rapide de la population a entrainé une croissance tout aussi rapide de la demande en eau de bonne qualité. Dans cette commune, les eaux usées domestiques sont déversées directement dans la nature sans aucun traitement préalable, suite à l'absence ou au dysfonctionnement des stations d'épuration existantes. Ces eaux sont susceptibles de polluer le sol et le sous-sol, et par la suite les eaux souterraines, en raison de leurs teneurs souvent élevées en matières organiques bio-dégradables et en microorganismes d'origine fécale. Le développement accéléré et incontrôlé de cette ville et la multiplication des quartiers dépourvus de tout plan d'urbanisation entraînent une prolifération de systèmes individuels d'assainissement inadéquats. L'infiltration, la persistance, la survie et l'évolution des microorganismes présents sont liées à tout un ensemble de facteurs comprenant notamment la nature du sol, les conditions environnementales prévalant dans ce sol et dans l'eau souterraine, et la qualité des eaux superficielles infiltrées. 
Cette commune qui a une faible couverture en approvisionnement en eau potable connaît par moments un phénomène d'inondation dont la conséquence est l'infiltration de l'eau de ruissellement qui contamine la nappe phréatique, voire les ouvrages hydrauliques. Cette nappe phréatique, ressource principale en eau de la commune, constitue le principal réservoir à partir duquel la majorité de la population est approvisionnée en eau de consommation. La présent travail s'intéresse à la recherche des facteurs associés à la qualité des eaux consommées dans la commune d'Abomey-Calavi au Bénin.

\section{Cadre d'étude et approche méthodologique}

\subsection{Présentation du cadre d'étude}

La commune d'Abomey-Calavi d'une superficie de $650 \mathrm{~km}^{2}$ soit 0,48 $\%$ de la superficie nationale, est située dans la partie sud de la République du Bénin et dans le département de l'Atlantique. Elle se trouve entre la latitude $6^{\circ} 20^{\prime} 23.4^{\prime \prime}$ et $6^{\circ} 42^{\prime} 6.6^{\prime \prime}$ nord et la longitude $2^{\circ} 14^{\prime} 13.8^{\prime \prime}$ et $2^{\circ} 25^{\prime} 7.8^{\prime \prime}$ est. Elle est limitée au Nord par la commune de Zè, au sud par l'océan Atlantique, à l'est par les communes de Sô-Ava, de Cotonou et à l'ouest par les communes de Tori-Bossito puis de Ouidah (figure1)

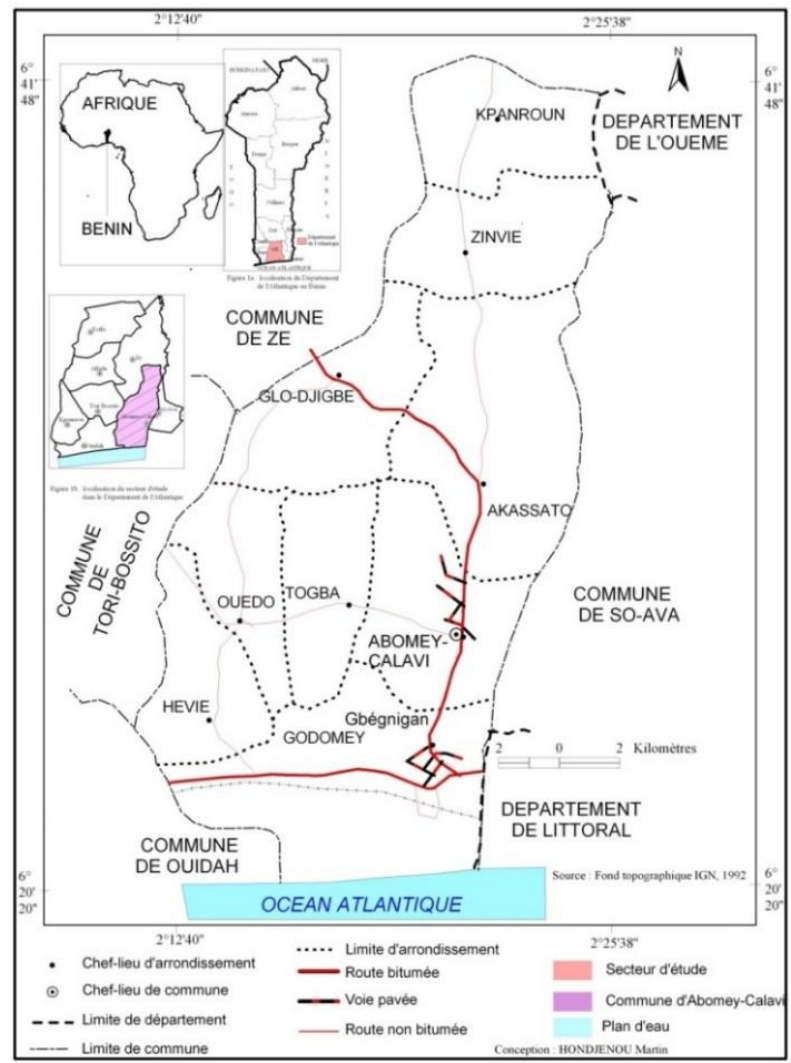

Figure 1 : Situation géographique de la commune d'Abomey-Calavi

Source : Résultats d'enquête, juillet 2017 


\subsection{Matériel et méthodes}

Dans le souci de collecter un maximum de données et d'informations fiables, plusieurs techniques ont été mises en œuvre sur le terrain.

\subsubsection{Méthode de collecte des données socio-anthropologiques}

Il s'agit d'une recherche transversale, descriptive et rétrospective sur les facteurs associés à la qualité de l'eau de consommation dans la commune d'Abomey-Calavi au Bénin. A cet effet 399 ménages ont été interviewés obtenus à partir de la formule de Beaud et Marien en 2003 avec un degré de confiance de $95 \%$ et une marge d'erreur de plus ou moins $5 \%$ et 30 personnes ressources choisies de façon aléatoire dans la commune d'Abomey-Calavi.

Les points d'eau à analyser ont été sélectionnés par choix raisonné tenant compte de leur répartition géographique. Le type de point d'eau a été un critère important dans la mesure où les puits de faible profondeur seraient plus exposés aux divers types de pollution que les puits profonds et les forages. Après observation et pré-enquête, il a été retenu trois niveaux pour les prélèvements que sont la source, le transport et le stockage à domicile. Ainsi, le nombre d'échantillons d'eau à prélever a été déterminé par la formule de SCHWARTZ (1995) suivant le protocole 2.

$$
\mathbf{N}=\mathbf{Z}^{2}(\mathbf{p})(\mathbf{1 - p}) \times \mathbf{F c} / \mathbf{I}^{2}
$$

Avec $\mathrm{N}$ : Taille des sources d'eau à prélever ;

$\mathrm{Z}$ : Taux de confiance de $95 \%(1,96)$;

$\mathrm{P}$ : Taux d'accès à l'eau dans le département de l'Atlantique en milieu rural est de $68,2 \%$;

I : Précision $(5 \%)$;

Fc : Facteur de correction (1).

Ainsi, 333,26 échantillons d'eau ont été dégagés auquel un taux (T) de $10 \%$ est appliqué pour avoir un échantillon opérationnel. L'échantillon $\mathrm{N}$ devient alors $\mathrm{N}_{\mathrm{f}}: \mathbf{N}_{\mathrm{f}}=\mathbf{N} \times \mathbf{1 0} \%$.

Un échantillon de 33 prélèvements d'eau a été donc retenu.

Les sites de prélèvement ont été choisis en tenant compte des paramètres morpho-structuraux, de l'accessibilité et de la pression humaine exercée sur les points d'eau. A la source, les échantillons ont été prélevés à la sortie du réseau. Pendant le transport, ils ont été faits dans tous les types de récipients rencontrés lors de l'enquête. Les échantillons faits au stockage de l'eau à domicile sont prélevés dans les jarres ou les plastiques qui servent de retenue d'eau pour les ménages.

Pour la collecte des données, cinq techniques ont été utilisées. Il s'agit de:

- l'enquête par questionnaire pour les ménages ;

- l'entretien individuel avec les responsables d'hygiène et d'assainissement du Centre de Santé et de la mairie de la Commune, 
le responsable de la Société Nationale des Eaux du Bénin et avec les chefs des villages enquêtés;

- l'entretien de groupe focalisé avec les femmes dans les villages enquêtés;

- l'observation directe des ménages et des alentours des sources d'approvisionnement en eau.

Les outils utilisés sont le questionnaire ménage, le guide d'entretien pour les personnes ressources et la grille d'observation.

Les matériels utilisés sont GPS (Global Positioning System) pour la prise des relevés de coordonnées géographiques des points d'eau, de l'appareil photographique numérique pour la prise de photos images visuelles parlantes devant servir à illustrer le développement de certains aspects de cette recherche

\subsubsection{Méthode de traitement des données}

Les données collectées sont traitées avec le logiciel Excel 2007. Etant donné que toutes les données collectées sont de nature qualitative et quantitative et sont principalement les sources, d'approvisionnement en eau, la qualité de l'eau et les facteurs qui influencent cette qualité. Ainsi, des croisements ont été faits pour juger de la significativité des liaisons entre ces paramètres et la morbidité hydrique. Il est calculé :

Prévalence $=\frac{\text { Nombre de cas }(\text { ancien }+ \text { nouveau }) \mathrm{d}^{\prime} \text { une maladie à un instant }(\mathrm{t})}{\text { Population cible à un instant } \mathrm{t}} X \mathbf{X} \mathbf{1 0 0 0}$

Ce paramètre renseigne sur l'importance d'une maladie ou d'une infection dans une population à un moment donné.

\section{Résultats et discussions}

\subsection{Source d'eau et usages ordinaires dans les ménages}

Dans la Commune d'Abomey-Calavi, l'eau est utilisée pour divers usages. Elle sert dans les activités de production ainsi que dans la consommation. La figure 2 montre les différentes sources d'eau et les usages qui sont faits de l'eau dans la Commune d'Abomey-Calavi. 


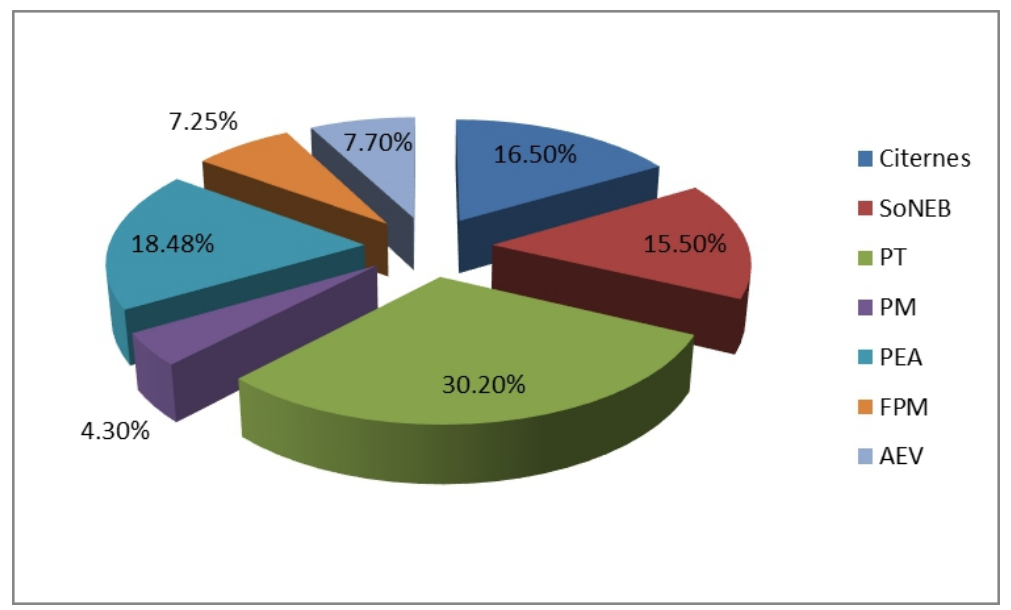

Figure 2 : Sources d'eau pour les usages domestiques Source : Travaux de terrain, juillet 2017

Il ressort de l'analyse de la figure 2 que la majorité des populations de la Commune d'Abomey-Calavi consomment l'eau des PT, des PEA, des citernes et de la SoNEB. Ainsi, dans la Commune d'Abomey-Calavi, pour les activités domestiques, la population utilise l'eau issue des forages (FPM, PEA, PM et $\mathrm{AEV}$ ) à $37,73 \%$, suivie de l'eau des puits traditionnels (30,20\%) et de l'eau des citernes à 16,50\%, L'eau de la SONEB n'est utilisée qu'à $15,50 \%$ et principalement pour la boisson. La lessive, la vaisselle, l'hygiène corporelle sont faites avec l'eau de pluie associée à l'eau des puits traditionnels.

\section{2 .Analyse bactériologique}

Les résultats des paramètres microbiologiques de l'eau des ouvrages prélevée à la source, au cours du transport et au moment du stockage sont présentés respectivement dans les tableaux I, II et III

Tableau I: Résultats des paramètres microbiologiques de l'eau prélevée à la source

\begin{tabular}{|c|c|c|c|c|c|c|}
\hline Paramètres & Normes OMS & PEA & AEV & FPM & Fleuve Togbo & PT \\
\hline Coliformes fécaux & $0 / 100 \mathrm{ml}$ & $0 / 100 \mathrm{ml}$ & $0 / 100 \mathrm{ml}$ & $0 / 100 \mathrm{ml}$ & $260 / 100 \mathrm{ml}$ & $156 / 100 \mathrm{ml}$ \\
\hline Coliformes totaux & $0 / 100 \mathrm{ml}$ & $0 / 100 \mathrm{ml}$ & $0 / 100 \mathrm{ml}$ & $47 / 100 \mathrm{ml}$ & $2380 / 100 \mathrm{ml}$ & $980 / 100 \mathrm{ml}$ \\
\hline Escherichia coli & $0 / 100 \mathrm{ml}$ & $0 / 100 \mathrm{ml}$ & $36 / 100 \mathrm{ml}$ & $12 / 100 \mathrm{ml}$ & $3200 / 100 \mathrm{ml}$ & $1400 / 100 \mathrm{ml}$ \\
\hline
\end{tabular}

Les résultats des paramètres microbiologiques mesurés dans l'eau au cours du transport sont présentés dans le tableau II.

Tableau II: Résultats des paramètres microbiologiques de l'eau prélevée au cours du transport

\begin{tabular}{|c|c|c|c|c|c|}
\hline Paramètres & $\begin{array}{c}\text { Normes } \\
\text { OMS }\end{array}$ & PEA & AEV & FPM & $\begin{array}{c}\text { Fleuve } \\
\text { Togbo }\end{array}$ \\
\hline Coliformes fécaux & $0 / 100 \mathrm{ml}$ & $17 / 100 \mathrm{ml}$ & $80 / 100 \mathrm{ml}$ & $30 / 100 \mathrm{ml}$ & $980 / 100 \mathrm{ml}$ \\
\hline Coliformes totaux & $0 / 100 \mathrm{ml}$ & $28 / 100 \mathrm{ml}$ & $938 / 100 \mathrm{ml}$ & $1600 / 100 \mathrm{ml}$ & $7110 / 100 \mathrm{ml}$ \\
\hline Escherichia coli & $0 / 100 \mathrm{ml}$ & $4 / 100 \mathrm{ml}$ & $65 / 100 \mathrm{ml}$ & $170 / 100 \mathrm{ml}$ & $12700 / 100 \mathrm{ml}$ \\
\hline
\end{tabular}

Source : Résultats des analyses du laboratoire DG-Eau, 2017 
La qualité bactériologique des échantillons d'eau prélevés au moment du stockage est présentée dans le tableau III.

Tableau III: Résultats des paramètres microbiologiques de l'eau prélevée au moment du stockage

\begin{tabular}{|c|c|c|c|c|c|c|}
\hline Paramètres & $\begin{array}{c}\text { Normes } \\
\text { OMS }\end{array}$ & PEA & AEV & FPM & $\begin{array}{c}\text { Fleuve } \\
\text { Togbo }\end{array}$ & PT \\
\hline Coliformes fécaux & $0 / 100 \mathrm{ml}$ & $780 / 100 \mathrm{ml}$ & $590 / 100 \mathrm{ml}$ & $600 / 100 \mathrm{ml}$ & $8820 / 100 \mathrm{ml}$ & $230 / 100 \mathrm{ml}$ \\
\hline Coliformes totaux & $0 / 100 \mathrm{ml}$ & $450 / 100 \mathrm{ml}$ & $1800 / 100 \mathrm{ml}$ & $4250 / 100 \mathrm{ml}$ & $28440 / 100 \mathrm{ml}$ & $\begin{array}{c}1100 / 100 \\
\mathrm{ml}\end{array}$ \\
\hline Escherichia coli & $0 / 100 \mathrm{ml}$ & $260 / 100 \mathrm{ml}$ & $870 / 100 \mathrm{ml}$ & $2400 / 100 \mathrm{ml}$ & $88900 / 100 \mathrm{ml}$ & $\begin{array}{c}2700 / 100 \\
\mathrm{ml}\end{array}$ \\
\hline
\end{tabular}

Source : Résultats des analyses du laboratoire DG-Eau, 2017

L'analyse microbiologique des échantillons d'eaux de puits, de forages et du fleuve Togbo a montré une forte présence de germes banals, de coliformes totaux et de germes pathogènes tels que les Escherichia coli, les Salmonella et Shigella, les streptocoques fécaux et les Clostridium. Les échantillons du fleuve Togbo et des puits contiennent les plus fortes concentrations de ces micro-organismes au-delà des valeurs maximales admissibles. Mais les échantillons de PEA à la sortie du réseau ne sont pas pollués par les germes pathogènes. Lors du transport et durant la conservation à domicile, ces eaux deviennent polluées par la présence des germes dont les valeurs sont au-dessus de celles admises par L'OMS. De ce point de vue, les eaux du milieu de recherche deviennent dans leur majorité polluées durant le transport et au stockage à domicile.

La présence de ces bactéries d'origine fécale dans l'eau de consommation indique une contamination de l'eau par des germes pathogènes comme Vibrio cholerae ou Salmonella Typhi (Lalêyè, 2016). L'infiltration du lixiviat au niveau de la nappe phréatique expose l'eau à diverses pollutions notamment celles bactériologiques. Selon Atchadé (2014), le nombre de germes bactériens varie surtout au niveau des forages pédestres dont le système de tête de pompe serait un facteur important d'infiltration des eaux souillées et contaminées donc d'accroissement de la charge polluante. En effet, cette partie de l'ouvrage est en contact permanent avec les pieds (parfois chargés de bactéries) des usagers de ces forages. Pour Mialo (2017), la présence en particulier d'Escherichia coli indique qu'il y a une insuffisance de protection de l'eau contre la pollution fécale dans les ménages. Cette contamination peut être corrélée au défaut d'hygiène des mains et du matériel de puisage. Plus les réservoirs sont de grands volumes, plus longtemps dure l'eau en stockage et plus grand est le nombre de contacts avec l'eau, impliquant plusieurs personnes. Dans ces circonstances, les réservoirs sont moins lavés avec un détergent et un désinfectant ; tous facteurs pouvant entraîner une sur-contamination de l'eau. 


\subsection{Analyse physico-chimique}

\subsubsection{Qualité physico-chimique de l'eau des ouvrages à la source}

Les résultats des paramètres physico-chimiques de l'eau des ouvrages prélevée à la source sont présentés dans le tableau IV.

Tableau IV: Résultats des paramètres physico-chimiques de l'eau prélevée à la source

\begin{tabular}{|c|c|c|c|c|c|c|}
\hline Paramètres & $\begin{array}{c}\text { Normes } \\
\text { ABeNor }\end{array}$ & PEA & AEV & FPM & $\begin{array}{c}\text { Puits } \\
\text { traditionnels }\end{array}$ & $\begin{array}{c}\text { Rivière } \\
\text { Togbo }\end{array}$ \\
\hline $\mathbf{p H}$ & $\begin{array}{c}6.5 \\
<\mathrm{ph}>8.5\end{array}$ & 6,705 & 6,959 & 6,58 & 6,74 & 6,95 \\
\hline $\begin{array}{c}\text { Conductivité } \\
(\boldsymbol{\mu S} / \mathbf{c m})\end{array}$ & 2100 & 55,4 & 56,3 & 73 & 450 & 687 \\
\hline TDS (mg/l) & - & 20 & 58,5 & 50 & 230 & 175 \\
\hline Couleur (PtCo) & 15 & 27 & 0 & 0,596 & 31 & 78 \\
\hline Turbidité(NTU) & 5 & 7 & 0 & 119,2 & 19 & 22,14 \\
\hline Nitrates (mg/l) & 45 & 1,6 & 12,76 & 0 & 31 & 9,8 \\
\hline Nitrites (mg/l) & 3,2 & 0,008 & 0,0264 & 0 & 1.28 & 1,01 \\
\hline Ammonium (mg/l) & 0,5 & 0,02 & 0,1161 & 1,0062 & 0.009 & 0,9 \\
\hline
\end{tabular}

Source : Résultats des analyses du laboratoire DG-Eau, 2017

Pour tous les éléments chimiques considérés, les valeurs obtenues pour les ouvrages sauf pour le fleuve Togbo sont toutes en dessous des normes de l'ABeNor (2003) et de l'OMS (2008). Les valeurs du pH des eaux de tous les ouvrages varient entre 5,705 et 6,85. Les résultats indiquent que la valeur du TDS varie dans l'ensemble des ouvrages de $20 \mathrm{mg} / 1$ pour les PEA à $230 \mathrm{mg} / 1$ pour les puits traditionnels en passant par des valeurs assez importantes de $58,5 \mathrm{mg} / \mathrm{l}$ pour les AEV, $50 \mathrm{mg} / \mathrm{l}$ pour les FPM et $175 \mathrm{mg} / 1$ pour le fleuve Togbo. Quant à la variabilité de la conductivité, elle montre des valeurs comprises entre 55,4 $\mu \mathrm{s} / \mathrm{cm}$ (PEA) et $687 \mu \mathrm{s} / \mathrm{cm}$ (fleuve Togbo).

Cette observation révèle une conformité à $100 \%$ des eaux analysées; car les critères normalisés béninois pour comparaison, limitent ce paramètre à $2100 \mu \mathrm{s} / \mathrm{cm}$. Le fleuve Togbo présente les valeurs les plus élevées pour tous les paramètres dosés. Sa couleur n'est pas claire car elle est $78 \mathrm{Pt}$ Co pour une norme de $15 \mathrm{Pt} \mathrm{Co,} \mathrm{trouble} \mathrm{car} \mathrm{elle} \mathrm{est} \mathrm{de} \mathrm{22,14} \mathrm{NTU} \mathrm{pour} \mathrm{une} \mathrm{norme} \mathrm{de} 5$ PTU, et chargée en ammonium car sa valeur est de $0,9 \mathrm{mg} / \mathrm{l}$ pour une norme de $0,5 \mathrm{mg} / \mathrm{l}$. Donc l'eau de la rivière est impropre à la consommation.

3.2.2. Qualité physico-chimique de l'eau des ouvrages lors du transport Les résultats des paramètres physico-chimiques mesurés dans l'eau au cours du transport sont présentés dans le tableau V.

Tableau V: Résultats des paramètres physico-chimiques prélevés durant le transport

\begin{tabular}{|c|c|c|c|c|c|}
\hline Paramètres & Normes OMS & PEA & AEV & FPM & Fleuve Togbo \\
\hline $\mathbf{p H}$ & $6.5<\mathrm{pH}>8.5$ & 6,05 & 6,28 & 6,37 & 6,90 \\
\hline $\begin{array}{c}\text { Conductivité } \\
(\boldsymbol{\mu S} / \mathbf{c m})\end{array}$ & 2100 & 153 & 202 & 225 & 359 \\
\hline TDS (mg/l) & - & 48 & 89 & 262 & 225 \\
\hline Couleur (PtCo) & 15 & 33 & 0,66 & 2,69 & 95 \\
\hline
\end{tabular}




\begin{tabular}{|c|c|c|c|c|c|}
\hline $\begin{array}{c}\text { Turbidité } \\
\text { (NTU) }\end{array}$ & 5 & 4,5 & 2,33 & 4,43 & 103,4 \\
\hline Nitrates (mg/l) & 45 & 6,8 & 15,5 & 0 & 17,9 \\
\hline Nitrites (mg/l) & 3,2 & 0,013 & 0,018 & 0,012 & 3,9 \\
\hline $\begin{array}{c}\text { Ammonium } \\
\text { (mg/l) }\end{array}$ & 0,5 & 0,03 & 0,8 & 1,23 & 2,6 \\
\hline
\end{tabular}

Source : Résultats des analyses du laboratoire DG-Eau, 2017

Lors du transport de l'eau prélevée, les valeurs du pH ont évolué pour les PEA, l'AEV, les FPM et le fleuve. Les résultats des TDS et de la conductivité montrent une variation. Les variations sont notées pour tous les ouvrages sauf pour l'AEV et le fleuve Togbo où ces valeurs ont plutôt baissé. L'eau des forages présente une valeur de turbidité assez élevée même si elle demeure en dessous de la norme ABeNor. Par contre, l'eau du fleuve Togbo est assez trouble car durant le transport sa valeur initiale de 22,4 NTU a évolué jusqu'à 103,4 NTU. Pour conclure, durant le transport, les valeurs des paramètres considérés ont vraiment augmentées pour certains. Mais, il faut noter que l'eau de la rivière présente les fortes valeurs de tous les paramètres dosés.

\subsubsection{Qualité physico-chimique de l'eau des ouvrages au stockage}

La qualité physico-chimique des échantillons d'eau prélevés au moment du stockage est présentée dans le tableau VI.

Tableau VI: Qualité physico-chimique des échantillons d'eau lors du stockage

\begin{tabular}{|c|c|c|c|c|c|c|}
\hline Paramètres & Normes OMS & PEA & AEV & FPM & $\begin{array}{c}\text { Puits } \\
\text { traditionnels }\end{array}$ & $\begin{array}{c}\text { Fleuve } \\
\text { Togbo }\end{array}$ \\
\hline $\mathbf{p H}$ & $6.5<\mathrm{pH}>8.5$ & 6,25 & 6,39 & 6,67 & 6,60 & 7,02 \\
\hline $\begin{array}{c}\text { Conductivité } \\
(\boldsymbol{\mu S} / \mathbf{c m})\end{array}$ & 2100 & 149,5 & 175 & 348 & 550,2 & 244 \\
\hline TDS (mg/l) & - & 123,5 & 109 & 149 & 25,3 & 223 \\
\hline Couleur (PtCo) & 15 & 2,14 & 5,37 & 0,01 & 10,5 & 166 \\
\hline Turbidité (NTU) & 5 & 2,26 & 3,71 & 1,11 & 1,26 & 56,70 \\
\hline Nitrates (mg/l) & 45 & 18,85 & 17,10 & 0 & 6,20 & 18,30 \\
\hline Nitrites (mg/l) & 3,2 & 0,16 & 0,15 & 0,024 & 0,018 & 0,019 \\
\hline Ammonium (mg/l) & 0,5 & 0,001 & 0,003 & 0,05 & 0,02 & 0,0044 \\
\hline
\end{tabular}

Source : Résultats des analyses du laboratoire DG-Eau, 2017

Le $\mathrm{pH}$ de tous les échantillons a évolué du transport au stockage d'eau à domicile. La conductivité par contre, a baissé dans tous les échantillons sauf au niveau des puits traditionnels même si elle est toujours assez considérable. Pour l'ensemble des paramètres chimiques, évalués, les valeurs obtenues sont assez faibles et restent inférieures à celles préconisées par l'OMS (2008) et l'ABeNOr (2003), sauf pour l'élément ammonium au niveau du fleuve Togbo, où sa teneur est $0,0044 \mathrm{mg} / \mathrm{l}$. Cette faible teneur en ammonium $\left(\mathrm{NH}_{4}{ }^{+}\right)$au niveau du fleuve Togbo montre que ces particules proviennent principalement 
du métabolisme cellulaire (Rejsek, 2002 ; Agassounon et al, 2010). Aussi, l'ammonium est un indicateur de la contamination des sources d'eau par la matière organique (Edorh et al 2010). En effet, en présence des microorganismes, l'azote organique contenu dans les matières organiques est oxydé en $\mathrm{NH}_{4}^{+}$. Les valeurs des paramètres physico-chimiques mesurées bien qu'elles soient aussi variées du transport au moment du stockage à domicile sont toutes en dessous des normes préconisées par l'ABénoR (2003) et l'OMS (2008). Les faibles teneurs en $\mathrm{NH}_{4}{ }^{+}$obtenues montrent que ces particules proviennent principalement du métabolisme cellulaire (Rejsek, 2002 ; Agassounon et al, 2010). Les nitrites ne sont retrouvés que rarement en concentrations importantes dans les eaux naturelles (Debieche, 2004), ce qui confirme les résultats de la présente étude. Seul l'ammonium dosé dans l'eau de la rivière présente une valeur au-dessus de la concentration maximale admise suivant les critères de comparaison. Ceci s'explique car, l'ammonium est un indicateur de la contamination des sources d'eau par la matière organique (Lalèyê, 2016). La nitrification convertit le $\mathrm{NH}_{4}{ }^{+}$en $\mathrm{NO}_{3}{ }^{-}$en passant par le $\mathrm{NO}_{2}$. Cette biotransformation s'effectue en présence de Nitrosomas et de Nitrobacter (Agassounon et al, 2010). Plusieurs auteurs Rejsek ; 2002; Zhang et al, 2008 et Lytle, et al, 2007 ont aussi révélé un lien entre les problèmes de corrosion et la nitrification.

Les nitrites ne sont retrouvés que rarement en concentrations importantes dans les eaux naturelles (Debieche, 2004), ce qui confirme les résultats de la présente étude.

\subsection{Facteurs associés à la qualité de l'eau de boisson}

Les tableaux VII et VIII présentent respectivement les facteurs associés à la qualité de l'eau de boisson en analyse uni variée et les facteurs associés à la qualité de l'eau (modèle final)

Le tableau VII présente les facteurs associés à la qualité de l'eau de boisson en analyse uni variée.

Tableau VII : Association entre la qualité de l'eau et les facteurs

\begin{tabular}{|c|c|c|c|c|}
\hline \multirow{2}{*}{$\begin{array}{l}\text { Variables } \\
\text { indépendantes }\end{array}$} & \multicolumn{2}{|c|}{ Qualité eau de boisson } & \multirow{2}{*}{$\begin{array}{l}\text { OR } \\
\text { [IC 95 \%] }\end{array}$} & \multirow[t]{2}{*}{ P-value } \\
\hline & $\begin{array}{l}\text { Mauvaise } \\
(\mathrm{n}=350)\end{array}$ & $\begin{array}{l}\text { Bonne } \\
(n=49)\end{array}$ & & \\
\hline $\begin{array}{c}\text { Type de source } \\
\text { d'approvisionnement } \\
\text { SoNEB } \\
\text { Eaux de surface } \\
\text { Ouvrages hydrauliques }\end{array}$ & $\begin{array}{l}4 \\
265 \\
81\end{array}$ & $\begin{array}{l}33 \\
16\end{array}$ & $\begin{array}{l}1 \\
173,33[16,05- \\
1871,60] \\
160,00[8,89-2280,46]\end{array}$ & $\begin{array}{l}\mathbf{0 , 0 0 0} \\
\mathbf{0 , 0 0 1}\end{array}$ \\
\hline
\end{tabular}




\begin{tabular}{|c|c|c|c|c|}
\hline $\begin{array}{l}\text { Récipient } \\
\text { manipulation eau } \\
\text { Propre } \\
\text { Sale }\end{array}$ & $\begin{array}{l}102 \\
248\end{array}$ & $\begin{array}{l}37 \\
12\end{array}$ & $\begin{array}{l}1 \\
7,32[1,03-19,30]\end{array}$ & 0,005 \\
\hline $\begin{array}{l}\text { Lavage des mains } \\
\text { Oui } \\
\text { Non }\end{array}$ & $\begin{array}{l}85 \\
265\end{array}$ & $\begin{array}{l}33 \\
16\end{array}$ & $\begin{array}{l}1 \\
6,19[1,69-22,64]\end{array}$ & 0,006 \\
\hline $\begin{array}{l}\text { Revenu mensuel } \\
<10000 \\
10000-30000 \\
>30000\end{array}$ & $\begin{array}{l}142 \\
98 \\
110 \\
\end{array}$ & $\begin{array}{l}4 \\
12 \\
33 \\
\end{array}$ & $\begin{array}{l}1 \\
0,23[0,22-2,33] \\
0,10[0,01-0,82]\end{array}$ & $\begin{array}{l}\mathbf{0 , 2 1 3} \\
\mathbf{0 , 0 3 2}\end{array}$ \\
\hline $\begin{array}{l}\text { Sensibilisation } \\
\text { Oui } \\
\text { Non }\end{array}$ & $\begin{array}{l}90 \\
260\end{array}$ & $\begin{array}{l}33 \\
16\end{array}$ & $\begin{array}{l}1 \\
5,81[1,59-21,23]\end{array}$ & $\mathbf{0 , 0 0 8}$ \\
\hline
\end{tabular}

Source : Traitement statistiques SPSS 20, octobre 2018

Le tableau VIII ci- dessous présente le résultat de l'analyse multi variée.

Tableau VIII : Facteurs associés à la qualité de l'eau (modèle final)

\begin{tabular}{|c|c|c|c|c|}
\hline \multirow[t]{2}{*}{ Variables indépendantes } & \multicolumn{2}{|c|}{ Qualité eau de boisson } & \multirow{2}{*}{$\begin{array}{c}\text { OR } \\
{[\text { IC } 95 \%]}\end{array}$} & \multirow[t]{2}{*}{$P$ value } \\
\hline & $\begin{array}{c}\text { Mauvaise } \\
(n=350)\end{array}$ & $\begin{array}{l}\text { Bonne } \\
(n=49)\end{array}$ & & \\
\hline $\begin{array}{c}\text { Récipient manipulation } \\
\text { eau } \\
\text { Propre } \\
\text { Sale }\end{array}$ & $\begin{array}{l}102 \\
248\end{array}$ & $\begin{array}{l}37 \\
12\end{array}$ & $\begin{array}{c}1 \\
9,94[1,05-94,22]\end{array}$ & 0,045 \\
\hline
\end{tabular}

Type de source

d'approvisionnement eau

SoNEB

Eaux de surface

Ouvrages hydrauliques

$\begin{array}{cc}207,68[13,07-3284,22] & \mathbf{0 , 0 0 0} \\ 234,29[8,75-6274,95] & \mathbf{0 , 0 0 1}\end{array}$

Source : Traitement statistiques SPSS 20, octobre 2018

Les enquêtés à $48,37 \%$ utilisent un récipient sale pour recueillir l'eau et cela n'est pas sans conséquence sur la qualité de l'eau. Ainsi l'eau peut être potable à la source et subir une pollution au cours du transport ou du stockage. Cette recherche a mis en évidence le lien entre l'état de propriété du récipient de puisage de l'eau et la qualité de l'eau de boisson $(\mathrm{p}=0,045)$. Le risque de contaminer l'eau est 7.32fois plus élevé chez les ménages qui utilisent un récipient de puisage sale que chez ceux qui utilisent un récipient propre.

Ces résultats sont en accord avec une étude menée par Claon et al en 2005 en Côte d'Ivoire qui a constaté que l'eau potable recueillie à la source par cette population est contaminée durant le stockage et les manipulations à domicile. 
Cette recherche a également montré un lien statistiquement significatif entre le type de source d'approvisionnement en eau utilisée $(p=0,000)$, le revenu mensuel $(\mathrm{p}=0,017)$, la sensibilisation de la population sur l'eau, l'hygiène et l'assainissement $(0,041)$ et la qualité de l'eau de boisson consommée dans les ménages. Des résultats similaires ont été obtenus dans une étude menée dans une commune rurale du Sénégal par Faye A et al, en 2011 avec une $\mathrm{p}<0,05$.

Le risque de contamination de l'eau est 173,33 fois plus élevé chez les ménages qui utilisent les puits traditionnels que chez ceux qui s'approvisionnent au robinet. Le risque de contamination de l'eau diminue de 10 fois chez les personnes qui ont un revenu élevé par rapport à celles qui ont un revenu faible. Le risque de contamination de l'eau est 5,81 fois plus élevé chez les personnes qui ne sont pas sensibilisées par rapport à celles qui sont sensibilisées. Le lavage des mains après défécation n'est pas pratiqué chez $70,41 \%$ des ménages. Cette recherche a mis en évidence un lien statistiquement associatif entre le non lavage des mains et la qualité de l'eau de boisson $(p=0,006)$. Ce résultat est en accord avec celui d'une étude faite dans le delta du Mékong au sud du Vietnam par Herbst S et al. en 2009. Cette recherche a montré que les mesures d'hygiène tel que le lavage des mains ne sont pas mises en pratique de manière intempestive dû à une conception erronée de risques et / ou à un manque de connaissances des relations entre eau et maladie ainsi que les habitudes ancrées.

Le risque de contaminer l'eau chez les personnes qui ne se lavent pas les mains après défécation est 6,19 fois plus élevé que chez ceux qui se lavent les mains.

Plusieurs autres facteurs pourraient expliquer une contamination de l'eau. Il s'agit entre autres de l'absence d'un système de collecte, de traitement et d'évacuation des ordures et des eaux usées, la défécation à l'air libre, la faible profondeur de la nappe phréatique (6 à $15 \mathrm{~m}$ selon les villages).

La présence de coliformes fécaux (Escherichia coli) est signe de contamination d'origine fécale et des mesures correctrices doivent être prises pour éviter les maladies hydriques.

\subsection{Facteurs non associés à la qualité de l'eau de boisson}

La taille de ménage n'est pas statistiquement associée à la qualité de l'eau de boisson. Ce résultat est contraire à une étude menée dans la commune de Klouékanmé au Benin par Agossou Y. en 2016. Cette différence pourrait s'expliquer par la taille de l'échantillon échantillon (399) qui est plus grande que celle de son étude (158).

Le niveau d'instruction n'est pas statistiquement associé la qualité de l'eau de boisson. Ce résultat est contraire à celui obtenu par Faye A et al, en 
2011 lors d'une étude réalisée dans une communauté rurale au Sénégal en 2011.

Dans cette recherche, le traitement de l'eau de boisson n'est pas statistiquement significatif à la qualité de l'eau. Son absence pourrait être une condition favorable au maintien en vie des germes pathogènes responsables des maladies.

\section{Conclusion}

Cette recherche a révélé que $67,76 \%$ des ménages ont une eau de mauvaise qualité dans la commune d'Abomey-Calavi. Aussi sur les 04 sources d'approvisionnement en eau des ménages prélevées 3 sont contaminées. Les facteurs associés à la qualité de l'eau de boisson sont l'état d'hygiène du récipient de manipulation de l'eau et le type de source d'approvisionnement en eau. La résolution de ce problème nécessite une bonne communication pour le changement de comportement de la communauté et un renforcement en infrastructure d'approvisionnement en eau potable.

\section{References:}

1. Agassounon Djikpo Tchibozo. M., Alassane A., Mama D., Ahanhanzo C., Toukourou F., et Agbangla C. (2010): Contrôle des paramètres physicochimiques des eaux en bouteille vendues à Cotonou. SOACHIM ; 7 : 131-138.

2. Agossou Y. (2009) : Facteurs associés à la qualité de l'eau de boisson dans les ménages de la commune de Klouékanmè au Bénin. Ouidah, IRSP, 78p.

3. Atchadé G. (2014): Impacts de la dynamique du climat et de l'occupation des terres sur les ressources en eau du bassin versant de la rivière zou dans le Bénin méridional. Thèse de Doctorat Unique. EDP/GENV/FLASH/UAC, 235 p.

4. CAE(2010) : Approvisionnement en eau potable au Bénin: problèmes et perspectives, Rapport ; 68 p.

5. Claon J.S., Sackou K.J., Attia A.R., et al., (2005) : Accessibilité, hygiène et qualité de l'eau à usage domestique dans la commune de Koumassi. Abidjan. Cahier de Santé Publique, pp 5-12.

6. Debieche T.H. (2004): Evolution de la qualité des eaux sous l'effet de la pollution saline, agricole et industrielle: La basse plaine Seybousse. Thèse de doctorat. Université Franche-Comté des sciences et techniques, $199 \mathrm{p}$.

7. Dégbey C., Makoutodé M., Ouendo E.M., Fayomi B., de Brouwer C. (2011) : Facteurs associés à la qualité de l'eau de puits et prévalence 
des maladies hydriques dans la commune d'Abomey-Calavi (Bénin). Santé 2011, volume 21, $\mathrm{n}^{\circ}$ 001, pp 47-55.

8. DG Eau (2010) : Evaluation du processus de professionnalisation de la gestion des AEV au Bénin, Rapport final, $131 \mathrm{P}$.

9. Edorh A. P., Adam S., Totin H., Koumolou L., Amoussou E., Aklikokou K. et Boko M. (2010) : Pesticides et métaux lourds dans l'eau de boisson, les sols et les sédiments de la ceinture cotonnière de Gogounou, Kandi et Banikoara (Bénin), Int. J. Biol. Chem. Sci., 4(4): 1170-1179.

10. Faye A, Ndiaye NM, Faye D, Tal-Dia A. (2011): Water quality and personal hygiene in rural areas of Senegal. Med Trop, pp 45-71.

11. INSAE (2013) : Effectifs de la population des villages et quartiers de ville du Bénin (RGPH-4, 2013), 85 p.

12. Herbst S, Benedikter S, Koester U, Phan N, Berger C, Rechenburg A. (2009): Perceptions of water, sanitation and health: a case study from the Mekong Delta, Vietnam, Water-SCI Technol, pp 699-707.

13. Lalêyè E.S. (2016) : Identification et analyse des déterminants de la contamination des eaux des sources et forages artésiens dans les communes de Zagnanado et de Zogbodomey au Bénin, Thèse pour l'obtention du diplôme de Doctorat Unique de l'Université d'AbomeyCalavi, EDP, FASHS, UAC, 269 p.

14. Lègba C. S. (2017) Croissance urbaine et accès à l'eau potable dans la ville de Djougou, Thèse pour l'obtention du diplôme de Doctorat Unique de l'Université d'Abomey-Calavi, EDP, FASHS, UAC, 224 p.

15. Lytle D., Sorg T., Wang L., Muhlen C., Rahrig M. et French, K. (2007): Biological nitrification in a full scale and pilot scale iron removal drinking water treatment plant. Journal Water SRT - Aqua; 56 (2): 125-136.

16. Mialo E. (2017) : Eau de boisson et maladies diarrhéiques dans la commune de Lalo au sud du Bénin. Thèse de doctorat, EDP/GENV/FASHS/UAC, 270 p.

17. OMS (2008): safer water, better health. Norme CCE de l'eau.

18. OMS (2016) : Trypanosomiase humaine africaine (maladie du sommeil) Aide-mémoire $\mathrm{N}^{\circ} 259$

19. Rejsek F. (2002): Analyse des eaux. Aspects réglementaires et techniques. Aquitaine, Bordeaux, France, 358 p.

20. Schwartz D. (1995) : Méthodes statistiques à l'usage des médecins et biologistes. Collection statistique en biologie et en médecine, 4ème édition, Flammarion, Médecine et Sciences, Paris, 314 p.

21. Sèbo E. (2014): Analyse des facteurs de différenciation de la morbidité diarrhéique dans le bassin géographique sud-Béninois du 
fleuve mono (Afrique de l'ouest). Thèse de doctorat, EDP/GENV/FLASH/UAC, $339 \mathrm{p}$.

22. Sohounou M. (2018): Qualité de l'eau de boisson et risques toxicologiques dans la commune d'Allada au Bénin, Thèse pour l'obtention du diplôme de Doctorat Unique de l'Université d'AbomeyCalavi, EDP, FASHS, UAC, 211 p.

23. Zhang Y., Groiffin A. et Edwards M. (2008): Nitrification in premise plumbing: role of phosphate, $\mathrm{pH}$, and pipe corrosion. Environ. Sci. Technol ; 42 (12) : 4280-4284. 